\title{
Zur Theorie der linearen Substitutionen. II.
}

\section{Von}

ATFRED LOEWY.

In einer unter dem gleichen Titel in diesen Annalen (Bd. 48, p. 97 ff.) veröffentlichten Arbeit, welche ich im Folgenden mit I citire, habe ich folgendes Theorem (p. 106) aufgestellt: Führen zuvei ähnliche lineare Substitutionen $U_{1}$ und $U_{2}$ dieselbe quadratische Form $S$ von nicht verschwindender Determinante kogredient in sich über, so können sie durch eine Substitution $R$, welche auch ebendieselbe quadratische Form $S$ in sich transformirt, in einander übergefiihrt werden.

$$
U_{1}=R U_{2} R^{-1} \text {. }
$$

Der Zweck der folgenden Zeilen ist es, einen neuen Beweis dieses Satzes zu geben. Die Methode dieses Beweises gilt in unveränderter Weise wie für die symmetrischen so auch für die alternirenden bilinearen Formen. Infolge dessen können genau analoge Betrachtungen, wie wir sie in I, \& 4 für die symmetrisehen Formen anstellten, jetzt auch für die alternirenden Formen durchgeführt werden. Hierdurch wird eine in Herrn Tabers Arbet "On the Automorphic Linear Transformation of an Alternate Bilinear Form ${ }^{* *}$ ) offen gelassene Frage, nämlich nach den nothwendigen und zugleich hinreichenden Bedingungen dafür, dass eine lineare Substitution, welche eine alternirende Form von nicht verschwindender Determinante kogredient in sich überführt, als Quadrat einer linearen Substitution, welche dieselbe alternirende Form in sich überführt, darstellbar ist, zur Entscheidung gebracht.

Seien $U_{1}$ and $U_{2}$ die Symbole für zwei lineare Substitutionen, welche beide dieselbe beliebige symmetrische [alternirende] bilineare Form $S$ von nicht verschwindender Determinante kogredient in sich transformiren; es sei:

$$
U_{1}^{\prime} S U_{1}=S ; \quad U_{2}^{\prime} S U_{2}=S .
$$

*) Math. Annalen, Bd. 46, Heft 4. 
Da $U_{1}$ und $U_{2}$ nach Voraussetzung ähnlich sind, so giebt es eine Substitution $\boldsymbol{P}$, dass:

$$
U_{2}=P^{-1} U_{1} P
$$

wird. Setzt man diesen Wert des $U_{2}$, sowie

$$
U_{2}^{\prime}=P^{\prime} U_{1}^{\prime}\left(P^{-1}\right)^{\prime}
$$

in die Gleichung:

so folgt:

$$
S=U_{2}^{\prime} S U_{2},
$$

Führt man:

$$
S=P^{\prime} U_{1}^{\prime}\left(P^{-1}\right)^{\prime} . S . P^{-1} U_{1} P .
$$

ein, so ergiebt sich:

$$
J_{1}^{\prime}=S U_{1}^{-1} S^{-1}
$$

Hieraus folgt:

$$
S=P^{\prime} S U_{1}^{-1} S^{-1} \cdot\left(P^{-1}\right)^{\prime} S P^{-1} U_{1} P .
$$

Bedeutet nun:

$$
U_{1}\left(P S^{-1} P^{\prime} S\right)=\left(P S^{-1} P^{\prime} S\right) \cdot U_{1} .
$$

$$
\chi\left(P S^{-1} P^{\prime} S\right)
$$

eine beliebige ganze Funktion von:

so wird:

$$
P S^{-1} P^{\prime} S,
$$

$$
\chi\left(P S^{-1} P^{\prime} S\right) U_{1}=U_{1} \chi\left(P S^{-1} P^{\prime} S\right)
$$

Führen wir mit Herrn Frobenius*) auch gebrochene Potenzen linearer Substitutionen ein, so ist die mit

$$
\left(P S^{-1} P^{\prime} S\right)^{\frac{1}{2}}
$$

bezeichnete Form auch eine ganze Funktion von

mithin wird :

$$
P S^{-1} P^{\prime} S
$$

$$
\left(P S^{-1} P^{\prime} S\right)^{\frac{1}{2}} U_{1}=U_{1}\left(P S^{-1} P^{\prime} S\right)^{\frac{1}{12}}
$$

Infolge dessen ergiebt sich, wenn

gesetzt wird:

$$
R=\left(P S^{-1} P^{\prime} S\right)^{-\frac{1}{2}} P
$$

$$
\begin{aligned}
R^{-1} U_{1} R & =P^{-1} \cdot\left(P S^{-1} P^{\prime} S\right)^{\frac{1}{2}} \cdot U_{1}\left(P S^{-1} P^{\prime} S\right)^{-\frac{1}{2}} P \\
& =P^{-1} U_{1} P=U_{2}, \\
V_{1} & =R U_{2} R^{-1} .
\end{aligned}
$$

Ich behaupte nun, die Substitution:

$$
R=\left(P S^{-1} P S\right)^{-\frac{1}{2}} P
$$

*) Frobenius, Ueber die cogredienten Transformationen der bilinearen Formen. Sitaungsberichte der Berliner Akademie, 16. Januar 1896. \$1. 
führt bei beliebiger Wahl von $\boldsymbol{P}$ die symmetrische [alternirende] Form $S$ cogredient in sich über; denn:

$$
\begin{aligned}
R^{\prime} S R & =P^{\prime}\left(S P S^{-1} P^{\prime}\right)^{-\frac{1}{2}} S\left(P S^{-1} P^{\prime} S\right)^{-\frac{1}{2}} P \\
& =\left(P^{\prime} S P S^{-1}\right)^{-\frac{1}{2}} \cdot P^{\prime} S P\left(S^{-1} P^{\prime} S P\right)^{-\frac{1}{2}} \\
& =\left(P^{\prime} S P S^{-1}\right)^{-\frac{1}{2}} \cdot\left(P^{\prime} S P S^{-1}\right) \cdot S\left(S^{-1} P^{\prime} S P\right)^{-\frac{1}{8}} \\
& =\left(P^{\prime} S P S-1\right)^{\frac{1}{2}} \cdot\left(P^{\prime} S P S^{-1}\right)^{-\frac{1}{2}} \cdot S \\
& =S .
\end{aligned}
$$

Hiermit ist unser Satz erwiesen. In dem Specialfall $S=E$ hat Herr Frobenius*):

$$
\left(P P^{\prime}\right)^{-\frac{1}{2}} \boldsymbol{P}
$$

als orthogonale Substitution angegeben.

Wenden wir uns nun zur ausschliesslichen Betrachtong der alternirenden Formen von nicht verschwindender Determinante. Es gilt zunächst folgender Satz:

Bildet man das Quadrat von allen nicht singulären Substitutionen, welche von der Form $\left.{ }^{* *}\right)$ :

$$
(S+Y)^{-1}(S-Y)
$$

sind, wo $Y$ eine beliebige symmetrische Form ist, und welche die gegebene alternirende Form $S$ von nicht verschwindender Determinante cogredient in sich überführen, so exhält man hierdurch von den singulären Sub. stitutionen, die $S$ in sich überführen, ausnahmslos alle diejenigen, bei denen die charakteristische Function die Elementartheiler $(\rho+1)^{\delta}$ paarweise besitat.

Der Beweis ist genan analog wie in I, § 4, p. 107 und 108 für symmetrische Formen zu führen. Es gelten bei alternirenden Formen folgende Thatsachen: Ist ein Product von Elementartheilern der Art vorgegeben, dass diese paarweise von gleichem Grade sind und für reeiproke Werthe Null werden, mit Ausnahme derer, welche für den Werth +1 oder -1 verschwinden und einen geraden Exponenten haben, so giebt es stets eine Substitution $P_{1}$ mit den vorgeschriebenen Elementartheilern der charakteristischen Function, welche eine alternirende Form $S_{1}$ von nicht verschwindender Determinante cogredient in sich überführt***). Ferner sind auch alle alternirenden Formen von nicht verschwindender Determinante congruent.

- Frobenins, a. a. 0. \$3.

*) Vgl. etwa Taber a. a. 0. $\$ 1$ in Bezug auf diese Formel.

**) Frobenius, Ueber lineare Substitutionen and bilineare Pormen. Journ. f. d. r. u. ang. Math Bd. 84, p. 41. 
Da unser Beweis auch für $\delta=0$ gilt, so finden wir auf nene Art das bereits von Herrn Taber*) ansgesprochene Resultat:

Die nicht singulären Substitutionen, welche von der Form:

$$
(S+Y)^{-1}(S-Y)
$$

sind, können ausnahmslos auch als Quadrate dargestellt werden.

Herr Taber*) theilt die Gesammtheit der linearen Substitutionen, welche eine alternirende Form $S$ von nicht verschwindender Determinante cogredient in sich überführen and welche eine Gruppe bilden, in zwei Arten ein. Er nennt eine Substitution von der ersten oder zweiten Art, je nachdem dieselbe als Quadrat einer linearen Substitution der Gruppe darstellbar ist oder sich dieser Darstellung entzieht.

Nach I, $\$ 3$ sind keine weiteren Substitutionen als diejenigen, deren charakteristische Function die Elementartheiler $(\rho+1)^{\delta}$ paarweise besitzt, [hierbei kann auch $\delta=0$ sein, d. h. die charakteristische Function hat keinen Elementartheiler $(\varrho+1)^{\delta}$ und wir haben eine nicht singuläre Substitution] als Quadrate nicht singulärer Substitutionen darstellbar.

Man zeigt anf dieselbe Art wie in I, $\$ 3$, dass durch Quadriren von singulären Substitutionen dieser Kreis auch nicht erweitert werden kann. Hieraus folgt:

Die nothwendige und hinreichende Bedingung dafür, dass eine lineare Substitution, welche eine alternirende Form $S$ von nicht verschwindender Determinante cogredient in sich überführt, nach der Taberschen Bezeichnung von der ersten Art ist, lautet: die charakteristische Function der linearen Substitutionen muss die Elementartheiler der Form $(\varphi+1)^{\delta}$ paarweise besitzen.

Ferner gelten folgende Sätze:

Durch das negative Quadrat von $(S+Y)^{-1}(S-Y)$ werden alle singulären Substitutionen dargestellt, welche die alternirende Form $S$ in sich ïberführen und deren charakteristische Function die Elementartheiler der Form $(\rho-1)^{\gamma}$ paarweise besitzt.

Das zuletzt angefübrte Resultat der Darstellung als negatives Quadrat gilt auch für $\gamma=0$; diese Substitutionen, welche Herr Frobenius in seiner grundlegenden Arbeit***) in der Form:

$$
(S+\bar{Y})^{-1}(Y-S)
$$

als Normaltypus angiebt und welche wir auch nach unserer in $I$ angewandten Terminologie als nicht singuläre Substitutionen bezeichnen, können nach der Taber'schen Bezeichnung von der zweiten Art sein; dies tritt ein, wenn die charakteristische Function der Substitution

*) Taber, a, a. 0. \$7.

**) Taber, a. a. $0 . \S 1$ und $\$ 7$.

***) Journ. f, d, r. o. ang. Math, Bd. 84, p. 37. 
einen nicht paarweise auftretenden Elementartheiler $(\varrho+1)^{\alpha}$, wo $\alpha>1$ ist, besitzt. $[\alpha=1$ ist unmöglich, denn dann würde der Elementartheiler $(\varphi+1)$ nach dem Frobenius'schen Fundamentalsatze*) paarweise auftreten.]

Mit unseren Hilfsmitteln beweist man auf neue Art den bereits von Herrn Taber ausgesprochenen Satz*):

Jede Substitution, welche eine alternirende Frorm $S$ von nicht verschwindender Determinante cogredient in sich überführt, ist stets als $2 m+1^{\text {te }}$ Potenz eines Substitution, wetche auch $S$ in sich transformirt, darstellbar; $m$ kann hierbei jeden beliebigen ganzzahligen Wert annehmen.

Rawitsch, im August 1896.

*) Journ. f. d. r. u. ang. Math. Bd. 84, p. 41.

**) Taber, a, a. 0. $\$ 7$.

In meiner Arbeit; I (Bd. 48) ist folgender Druckfehler za verbessern: p. 104 Zeile 14 lies: $-\nabla+\varrho E$ statt $\nabla+\varrho E$. 